\title{
INTERACTION OF A SHORT-WAVE FIELD WITH A DOMINANT LONG WAVE IN DEEP WATER: DERIVATION FROM ZAKHAROV'S SPECTRAL FORMULATION
}

\author{
A. D. D. CRAIK
}

(Received 6 May, 1987; revised 2 August 1987)

\begin{abstract}
The leading-order interaction of short gravity waves with a dominant long-wave swell is calculated by means of Zakharov's [7] spectral formulation. Results are obtained both for a discrete train of short waves and for a localised wave packet comprising a spectrum of short waves.

The results for a discrete wavetrain agree with previous work of LonguetHiggins \& Stewart [5], and general agreement is found with parallel work of Grimshaw [4] which employed a very different wave-action approach.
\end{abstract}

\section{Introduction}

The quadratic and cubic interactions among a spectrum of deep-water gravity waves were represented by Zakharov [7] in terms of an integro-differential equation. This formulation was later adopted and expounded in greater detail by Crawford, Saffman \& Yuen [2], Crawford, Lake, Saffman \& Yuen [3] and Yuen \& Lake [6]; see also Craik [1] for a brief account. Not surprisingly, solutions of Zakharov's equations are available only for special cases, but these known solutions often improve upon previous approximations. For example, the evolution of a weakly-nonlinear two-dimensional wavetrain centred on a single dominant frequency is more accurately represented by the Zakharov method than by a nonlinear Schrödinger equation; and the 'Type 1' two- and three-dimensional modulational instabilities of periodic wavetrains are better described than by

\footnotetext{
${ }^{1}$ Mathematical Institute, University of St. Andrews, St. Andrews Fife KY16 9SS, Scotland.

(C) Copyright Australian Mathematical Society 1988, Serial-fee code 0334-2700/88
} 
the Benjamin-Feir and Phillips resonance models; see Yuen and Lake [6] for details.

However, no study has yet been undertaken on the interaction of short and long waves from the Zakharov standpoint. Such a study provides an interesting complement to an approach via wave action by Grimshaw [4], which developed in parallel with the present work. Because of the algebraic complexity that underlies the simplicity of Zakharov's formulation, only a leading-order approximation is discussed here. Thus, only quadratic interactions of long and short waves are considered, much as Longuet-Higgins and Stewart [5] first did for discrete modes. In this limiting case, the leading-order result of Longuet-Higgins and Stewart is recovered, with the ratio of wavenumbers of long and short waves as governing parameter. Also, results are obtained for localised packets of short waves modified by a long-wave 'swell', that agree at leading order with the findings of Grimshaw's analysis based on wave action. The analysis described here is of course capable of extension to higher orders of approximation, both in terms of the characteristic wavenumber ratio and to incorporate cubic resonant and nearresonant interactions. However, the algebraic complexity of doing so should not be under-estimated!

\section{General formulation}

To avoid elaborate preamble, we adopt the notation and formulation of Yuen and Lake [6, pp. 111-6]. As only quadratic interactions need be considered, the governing integro-differential equation is (c.f. Yuen and Lake, equation 107)

$$
\begin{aligned}
& b_{t}-i \omega b+i \iint_{-\infty}^{\infty} V^{(1)}\left(\mathbf{k}, \mathbf{k}_{1}, \mathbf{k}_{2}\right) b\left(\mathbf{k}_{1}\right) b\left(\mathbf{k}_{2}\right) \delta\left(\mathbf{k}-\mathbf{k}_{1}-\mathbf{k}_{2}\right) d \mathbf{k}_{1} d \mathbf{k}_{2} \\
& \quad+i \iint_{-\infty}^{\infty} V^{(2)}\left(\mathbf{k}, \mathbf{k}_{1}, \mathbf{k}_{2}\right) b^{*}\left(\mathbf{k}_{1}\right) b\left(\mathbf{k}_{2}\right) \delta\left(\mathbf{k}+\mathbf{k}_{1}-\mathbf{k}_{2}\right) d \mathbf{k}_{1} d \mathbf{k}_{2} \\
& \quad+i \iint_{-\infty}^{\infty} V^{(3)}\left(\mathbf{k}, \mathbf{k}_{1}, \mathbf{k}_{2}\right) b^{*}\left(\mathbf{k}_{1}\right) b^{*}\left(\mathbf{k}_{2}\right) \delta\left(\mathbf{k}+\mathbf{k}_{1}+\mathbf{k}_{2}\right) d \mathbf{k}_{1} d \mathbf{k}_{2} \\
& \quad+\cdots=\mathbf{0}
\end{aligned}
$$

where the complex function $b(\mathbf{k}, t)$ is defined, in terms of the complex Fourier transforms $\hat{\eta}(\mathbf{k}, t)$ and $\hat{\phi}^{s}(\mathbf{k}, t)$ of the free-surface elevation and free-surface velocity potential respectively, as

$$
b(\mathbf{k}, t)=[\omega(\mathbf{k}) / 2|\mathbf{k}|]^{1 / 2} \hat{\eta}(\mathbf{k}, t)+i[|\mathbf{k}| / 2 \omega(\mathbf{k})]^{1 / 2} \hat{\phi}^{s}(\mathbf{k}, t) .
$$

The interaction coefficients $V^{(i)}\left(\mathbf{k}, \mathbf{k}_{1}, \mathbf{k}_{2}\right)$ are lengthy algebraic expressions, given incorrectly in Yuen and Lake's Appendix A but correctly in Appendix 
A of Crawford et al. [2], as

$$
\begin{gathered}
V^{(1)}\left(\mathbf{k}, \mathbf{k}_{1}, \mathbf{k}_{2}\right)=V_{0, \mathbf{1}, 2}^{(-)}, \quad V^{(2)}\left(\mathbf{k}, \mathbf{k}_{1}, \mathbf{k}_{2}\right)=2 V_{2,0,1}^{(-)}, \quad V^{(3)}\left(\mathbf{k}, \mathbf{k}_{1}, \mathbf{k}_{2}\right)=V_{0,1,2}^{(+)} \\
V_{0,1,2}^{( \pm)} \equiv \frac{1}{8 \pi \sqrt{2}}\left\{\left(\mathbf{k} \cdot \mathbf{k}_{1} \pm k k_{1}\right)\left[\frac{\omega \omega_{1} k_{2}}{\omega_{2} k k_{1}}\right]^{1 / 2}+\left(\mathbf{k} \cdot \mathbf{k}_{2} \pm k k_{2}\right)\left[\frac{\omega \omega_{2} k_{1}}{\omega_{1} k k_{2}}\right]^{1 / 2}\right. \\
\left.+\left(\mathbf{k}_{1} \cdot \mathbf{k}_{2}+\dot{k}_{1} \dot{k}_{2}\right)\left[\frac{\omega_{1} \omega_{2} k}{\omega k_{1} k_{2}}\right]^{1 / 2}\right\}
\end{gathered}
$$

Throughout, superscript $*$ denotes complex conjugate and the frequencies $\omega(\mathbf{k})$ are given by the linear dispersion relation $\omega(\mathbf{k})=(g k)^{1 / 2}$ where $k \equiv|\mathbf{k}|$.

In previous applications of Zakharov's formulation, the quadratic interactions played a subsidiary role and the cubic resonant interactions provided the dominant wave-coupling mechanism. Here, in constrast, the disparate wavelengths of long and short waves allow quadratic interactions to dominate cubic ones; this invalidates the assumption, made in previous applications, that the periodicities of quadratic interactions are all far from those of linear waves.

To study the interaction of a continuous spectrum of (relatively) short waves with a single dominant long wave, one may set

$$
b(\mathbf{k}, t)=B(\mathbf{k}, t) \exp [-i \omega(\mathbf{k}) t]+\text { h.o.t., }
$$

where

$$
B(\mathbf{k}, t)=B_{w} \delta\left(\mathbf{k}-\mathbf{k}_{w}\right)+\hat{B}(\mathbf{k}, t),
$$

and the short-wave spectrum $\hat{B}(\mathbf{k}, t)$ is regarded as nonzero only for a range of wavenumbers $\mathbf{k}$ that are much larger than the wavenumber $\mathbf{k}_{w}$ of the long wave. Here, $\delta\left(\mathbf{k}-\mathbf{k}_{w}\right)$ denotes the Dirac delta function and $B_{w}$ may be taken to be a complex constant to the required order of approximation. It follows (c.f. Yuen and Lake, equation 109) that

$$
\begin{aligned}
i \partial B / \partial t= & \iint_{-\infty}^{\infty} V_{012}^{(1)} B_{1} B_{2} \delta_{0-1-2} \exp \left[i\left(\omega-\omega_{1}-\omega_{2}\right) t\right] d \mathbf{k}_{1} d \mathbf{k}_{2} \\
& +\iint_{-\infty}^{\infty} V_{012}^{(2)} B_{1}^{*} B_{2} \delta_{0+1-2} \exp \left[i\left(\omega+\omega_{1}-\omega_{2}\right) t\right] d \mathbf{k}_{1} d \mathbf{k}_{2} \\
& +\iint_{-\infty}^{\infty} V_{012}^{(3)} B_{1}^{*} B_{2}^{*} \delta_{0+1+2} \exp \left[i\left(\omega+\omega_{1}+\omega_{2}\right) t\right] d \mathbf{k}_{1} d \mathbf{k}_{2}
\end{aligned}
$$

where $\delta_{0-1-2}=\delta\left(\mathbf{k}-\mathbf{k}_{1}-\mathbf{k}_{2}\right), V_{012}^{(i)}=V^{(i)}\left(\mathbf{k}, \mathbf{k}_{1}, \mathbf{k}_{2}\right), B_{i}=B\left(\mathbf{k}_{i}, t\right)$, etc.

As short waves are assumed of sufficiently small amplitude that their interaction with the long wave dominates their mutual interactions, (2.6) simplifies 
to

$$
\begin{aligned}
i \partial \hat{B} / \partial t= & B_{w} \hat{B}\left(\mathbf{k}-\mathbf{k}_{w}\right) A \exp \left[i t\left(\omega-\omega_{w}-\omega\left(\mathbf{k}-\mathbf{k}_{w}\right)\right)\right] \\
& +B_{w}^{*} \hat{B}\left(\mathbf{k}+\mathbf{k}_{w}\right) B \exp \left[i t\left(\omega+\omega_{w}-\omega\left(\mathbf{k}+\mathbf{k}_{w}\right)\right)\right] \\
& +B_{w} \hat{B}^{*}\left(\mathbf{k}_{w}-\mathbf{k}\right) C \exp \left[i t\left(\omega-\omega_{w}+\omega\left(\mathbf{k}_{w}-\mathbf{k}\right)\right)\right] \\
& +B_{w}^{*} \hat{B}^{*}\left(-\mathbf{k}-\mathbf{k}_{w}\right) D \exp \left[i t\left(\omega+\omega_{w}+\omega\left(-\mathbf{k}-\mathbf{k}_{w}\right)\right)\right]
\end{aligned}
$$

with interaction coefficients $A, B, C, D$ defined as

$$
\begin{aligned}
A & \equiv V^{(1)}\left(\mathbf{k}, \mathbf{k}_{w}, \mathbf{k}-\mathbf{k}_{w}\right)+V^{(1)}\left(\mathbf{k}, \mathbf{k}-\mathbf{k}_{w}, \mathbf{k}_{w}\right), \quad B \equiv V^{(2)}\left(\mathbf{k}, \mathbf{k}_{w}, \mathbf{k}+\mathbf{k}_{w}\right) \\
C & \equiv V^{(2)}\left(\mathbf{k}, \mathbf{k}_{w}-\mathbf{k}, \mathbf{k}_{w}\right) \\
D & \equiv V^{(3)}\left(\mathbf{k}, \mathbf{k}_{w},-\mathbf{k}-\mathbf{k}_{w}+V^{(3)}\left(\mathbf{k},-\mathbf{k}-\mathbf{k}_{w}, \mathbf{k}_{w}\right) .\right.
\end{aligned}
$$

From the expressions for $V_{0,1,2}^{( \pm)}\left(\mathbf{k}, \mathbf{k}_{1}, \mathbf{k}_{2}\right)$ given by Crawford et al. it is found that good approximations are

$$
\begin{aligned}
& A=\frac{\left(\mathbf{k} \cdot \mathbf{k}_{w}\right)}{4 \pi}\left[\frac{2 \omega_{w}}{k_{w}}\right]^{1 / 2}\left[1+0\left[\frac{k_{w}}{k}\right]\right], \quad B=\frac{\left(\mathbf{k} \cdot \mathbf{k}_{w}\right)}{2 \pi}\left[\frac{2 \omega_{w}}{k_{w}}\right]^{1 / 2)}\left[1+O\left[\frac{k_{w}}{k}\right]\right], \\
& C=-\frac{k k_{w}}{4 \pi}\left[\frac{2 \omega_{w}}{k_{w}}\right]^{1 / 2}\left[1+0\left[\frac{k_{w}}{k}\right]\right], \quad D=\frac{k k_{w}}{4 \pi}\left[\frac{2 \omega_{w}}{k_{w}}\right]^{1 / 2}\left[1+O\left[\frac{k_{w}}{k}\right]\right] .
\end{aligned}
$$

The free-surface elevation $\eta(\mathbf{x}, t)$ may be recovered (c.f. Yuen and Lake, equation 106) from

$$
\begin{gathered}
\eta(\mathbf{x}, t)=\mathscr{b}(\mathbf{x}, t)+\mathscr{b}^{*}(\mathbf{x}, t) \\
\mathscr{b}(\mathbf{x}, t) \equiv \frac{B_{w}}{2 \pi}\left[\frac{k_{w}}{2 \omega_{w}}\right]^{1 / 2} e^{i\left(\mathbf{k}_{w} \cdot \mathbf{x}-\omega_{w} t\right)}+\frac{1}{2 \pi} \int_{-\infty}^{\infty}\left[\frac{k}{2 \omega(\mathbf{k})}\right]^{1 / 2} \hat{B}(\mathbf{k}, t) e^{-\imath \omega t+i \mathbf{k} \cdot \mathbf{x}} d \mathbf{k} .
\end{gathered}
$$

In the following sections, approximate particular solutions of (2.7) are given for cases of (i) discrete short waves, as in the analysis of Longuet-Higgins and Stewart [5], and (ii) a continuous but fairly narrow spectrum of short waves centred on wavenumber $\mathbf{k}_{0}$ and with a characteristic spectral width $\Delta$ that satisfies $k_{w} \ll \Delta \ll \mathrm{k}_{0}$. The latter case is exemplified by calculation of the modulations of a particular finite wave packet as it is traversed by the long wave.

\section{Discrete interactions}

To gain an understanding of the Zakharov formulation, it is helpful first to recover Longuet-Higgins and Stewart's [5] results for interaction of discrete modes. Accordingly, it is here further assumed that the short-wave field is dominated by a single Fourier mode. Then,

$$
\hat{B}(\mathbf{k}, t)=\hat{B}_{0} \delta\left(\mathbf{k}-\mathbf{k}_{0}\right)+\hat{\hat{B}}(\mathbf{k}, t)
$$


where $\hat{\hat{B}}$ represents the modulations due to quadratic interaction of $\hat{B}_{0}$ with the long wave. It follows from (2.7) that $\hat{\hat{B}}$ satisfies

$$
\begin{aligned}
i \partial \hat{\hat{B}} / \partial t= & B_{w} \hat{B}_{0} \delta\left(\mathbf{k}-\mathbf{k}_{w}-\mathbf{k}_{0}\right) A \exp \left[i t\left(\omega-\omega_{w}-\omega\left(\mathbf{k}-\mathbf{k}_{w}\right)\right)\right] \\
& +B_{w}^{*} \hat{B}_{0} \delta\left(\mathbf{k}+\mathbf{k}_{w}-\mathbf{k}_{0}\right) B \exp \left[i t\left(\omega+\omega_{w}-\omega\left(\mathbf{k}+\mathbf{k}_{w}\right)\right)\right] \\
& +B_{w} \hat{B}_{0}^{*} \delta\left(\mathbf{k}_{w}-\mathbf{k}_{0}-\mathbf{k}\right) C \exp \left[i t\left(\omega-\omega_{w}+\omega\left(\mathbf{k}_{w}-\mathbf{k}\right)\right)\right] \\
& +B_{w}^{*} \hat{B}_{0}^{*} \delta\left(-\mathbf{k}-\mathbf{k}_{w}-\mathbf{k}_{0}\right) D \exp \left[i t\left(\omega+\omega_{w}+\omega\left(-\mathbf{k}-\mathbf{k}_{w}\right)\right)\right]+\text { h.o.t. }
\end{aligned}
$$

Notice that the first two terms are slowly varying in time but that the last two vary rapidly, with frequency close to $2 \omega(\mathbf{k})$. This equation may be integrated directly to give

$$
\begin{aligned}
i \hat{\hat{B}}(\mathbf{k}, t)= & \frac{B_{w} \hat{B}_{0} \delta\left(\mathbf{k}-\mathbf{k}_{w}-\mathbf{k}_{0}\right) A \exp \left[i t\left(\omega-\omega_{w}-\omega\left(\mathbf{k}-\mathbf{k}_{w}\right)\right)\right]}{i\left[\omega-\omega_{w}-\omega\left(\mathbf{k}-\mathbf{k}_{w}\right)\right]} \\
& +\frac{B_{w}^{*} \hat{B}_{0} \delta\left(\mathbf{k}+\mathbf{k}_{w}-\mathbf{k}_{0}\right) B \exp \left[i t\left(\omega+\omega_{w}-\omega\left(\mathbf{k}+\mathbf{k}_{w}\right)\right)\right]}{i\left[\omega+\omega_{w}-\omega\left(\mathbf{k}+\mathbf{k}_{w}\right)\right]} \\
& +\frac{B_{2} \hat{B}_{0}^{*} \delta\left(\mathbf{k}_{w}-\mathbf{k}_{0}-\mathbf{k}\right) C \exp \left[i t\left(\omega-\omega_{w}+\omega\left(\mathbf{k}_{w}-\mathbf{k}\right)\right)\right]}{i\left[\omega-\omega_{w}+\omega\left(\mathbf{k}_{w}-\mathbf{k}\right)\right]} \\
& +\frac{B_{w}^{*} B_{0}^{*} \delta\left(-\mathbf{k}-\mathbf{k}_{w}-\mathbf{k}_{0}\right) D \exp \left[i t\left(\omega+\omega_{w}+\omega\left(-\mathbf{k}-\mathbf{k}_{w}\right)\right)\right]}{i\left[\omega+\omega_{w}+\omega\left(-\mathbf{k}-\mathbf{k}_{w}\right)\right]} \\
& +\cdots .
\end{aligned}
$$

Note that, owing to the respective denominators, which are $O\left(\omega_{w}\right)$ and $O(\omega)$, the rapidly-oscillating terms have amplitudes smaller by a factor $O\left[\left(k_{w} / k_{0}\right)^{1 / 2}\right]$. This will be exploited in the next section. An arbitrary constant of integration has been omitted without loss.

Fourier synthesis in (2.10) yields the surface elevation

$$
\begin{aligned}
\eta(x, t) \cong & \frac{\left|B_{w}\right|}{\pi}\left[\frac{k_{w}}{2 \omega_{w}}\right]^{1 / 2} \cos \left(k_{w} x-\omega_{w}+t+\theta_{w}\right)+\frac{\left|\hat{B}_{0}\right|}{\pi}\left[\frac{k_{0}}{2 \omega_{0}}\right]^{1 / 2} \cos \left(k_{0} x-\omega_{0}+\theta_{0}\right) \\
& -\frac{2\left|B_{w}\right|\left|\hat{B}_{0}\right| A}{\pi}\left[\frac{k_{0}}{2 \omega_{0}}\right]^{1 / 2}\left[\frac{\omega_{0+w} \cos \left[\left(k_{0}+k_{w}\right) x-\left(\omega_{0}+\omega_{w}\right) t+\theta_{w}+\theta_{0}\right]}{\omega_{0+w}^{2}-\left(\omega_{0}+\omega_{w}\right)^{2}}\right. \\
& \left.+\frac{\left(\omega_{w}-\omega_{0}\right) \cos \left[\left(k_{0}-k_{w}\right) x-\left(\omega_{0}-\omega_{w}\right) t+\theta_{0}-\theta_{w}\right]}{\omega_{0-w}^{2}-\left(\omega_{0}-\omega_{w}\right)^{2}}\right]+\cdots,
\end{aligned}
$$

after some reduction. In the latter, $\theta_{w}$ and $\theta_{0}$ denote the constant phases of $B_{w}$ and $\hat{B}_{0}$ respectively. The short notation $\omega_{0}=\omega\left(\mathbf{k}_{0}\right), \omega_{w}=\omega\left(\mathbf{k}_{w}\right)$ and $\omega_{0 \pm w}=$ $\omega\left(\mathbf{k}_{\mathbf{0}} \pm \mathbf{k}_{w}\right)$ has been adopted. The first two terms denote the unmodulated long and short waves while the remaining terms describe their interaction. For simplicity, attention is further restricted to unidirectional wavenumbers, taken 
along the $x$-direction, and leading-order approximations (2.9) to $A, B, C, D$ are expressed in terms of

$$
A \equiv \frac{\sqrt{2}}{4 \pi} k_{0} k_{w}\left[\omega_{w} / k_{w}\right]^{1 / 2} .
$$

The leading-order approximation in $k_{w} / k_{0}$ is easily recovered by Taylor-series expansion of $\omega_{0 \pm w}$. This is

$$
\begin{aligned}
\eta(x, t)= & a_{w} \cos \left(k_{w} x-\omega_{w} t+\theta_{w}\right)+a_{0} \cos \left(k_{0} x-\omega_{0} t+\theta_{0}\right) \\
& -a_{0} a_{w} k_{0} \sin \left(k_{0} x-\omega_{0} t+\theta_{0}\right) \sin \left(k_{w} x-\omega_{w} t+\theta_{w}\right),
\end{aligned}
$$

with relative error $0\left(k_{w} / k\right)$, where

$$
a_{w}=\frac{\left|B_{w}\right|}{\pi}\left[\frac{k_{w}}{2 \omega_{w}}\right]^{1 / 2}, \quad a_{0}=\frac{\left|\hat{B}_{0}\right|}{\pi}\left[\frac{k_{0}}{2 \omega_{0}}\right]^{1 / 2}
$$

denote the linear wave amplitudes. This result agrees with the result of LonguetHiggins and Stewart [5, eqs. 2.22 to 2.26] that the leading-order effect is a local change in the wavenumber of the short wave to $k^{\prime}=k_{0}+\delta k$, where

$$
\delta k=a_{w} k_{w} k_{0} \cos \left(k_{w} x-\omega_{w} t+\theta_{w}\right) .
$$

Note, however, that the local change in amplitude, in the present notation, is

$$
a^{\prime}-a_{0}=a_{0} a_{w} k_{w} \cos \left(k_{w} x-\omega_{w} t+\theta_{w}\right)
$$

according to Longuet-Higgins \& Stewart's equation (2.27); but this is not recovered by the present theory at this order of approximation. This apparently paradoxical result is due to the fact that the leading-order term (Longuet-Higgins and Stewart's term in $Q$ ) is 90 degrees out of phase with the original wave, whereas the next-order term in $k_{w} / k_{0}$ in the above would give their term in $P$, which has the same phase as the original wave. Since the change in amplitude is more easily derived from the wave-action equation, once the change (3.6) in wavenumber is known, this higher-order calculation is not presented.

\section{Interactions of a continuous spectrum}

Equation (2.7) may be used as starting point, with $\hat{B}(k, t)$ taken as a continuous, two-dimensional, short-wave spectrum centred on some wave-number $k_{0}$. For simplicity, it is assumed that the spectral bandwith, say $\Delta$, is small compared with $k_{0}$ but large compared with $k_{w}$. The latter restriction enables $\hat{B}\left(k \pm k_{w}\right)$ and $\omega\left(k \pm k_{w}\right)$ to be approximated by their Taylor expansions

$$
\begin{aligned}
\hat{B}\left(k \pm k_{w}, t\right) & =\hat{B}(k, t) \pm k_{w} \hat{B}^{\prime}(k, t)+\cdots, \\
\omega\left(k \pm k_{w}\right) & =\omega(k) \pm k_{w} \omega^{\prime}(k)+\cdots,
\end{aligned}
$$


where the prime ( $)^{\prime}$ denotes partial differentiation with respect to $k$. It follows that

$$
\begin{aligned}
\partial \hat{B} / \partial t=a_{w} \omega_{w} k\{ & -i \hat{B} \cos \left[t\left(\omega_{w}-k_{w} \omega^{\prime}\right)-\theta_{w}\right] \\
& +k_{w}(\partial \hat{B} / \partial k) \sin \left[t\left(\omega_{w}-k_{w} \omega^{\prime}\right)-\theta_{w}\right] \\
& \left.\left.+ \text { (rapidly-varying terms in } \exp ^{ \pm 2 i \omega t}\right)\right\},
\end{aligned}
$$

on using $2 A\left|B_{w}\right|=a_{w} \omega_{w} k$, where $a_{w}$ is the long-wave amplitude.

As in the discrete case described above, the rapidly-varying terms in (4.2) contribute to $\hat{B}(k, t)$ at higher order in $k_{0} / k_{w}$ than the slowly varying ones. These may therefore be ignored in seeking the leading-order approximation; experience of the discrete case indicates that the relative error incurred is $0\left(k_{w} / k_{0}\right)$. With the change to dimensionless quantities

$$
\delta \equiv a_{w} k_{w}\left[1-\frac{k_{w} \omega^{\prime}}{\omega_{w}}\right]^{-1}, \quad \kappa \equiv \delta k / k_{w}, \quad \tau \equiv\left(\omega_{w}-k_{w} \omega\right), t-\theta_{w},
$$

(4.2) becomes

$$
\partial \hat{B} / \partial \tau-(\delta \sin \tau) \kappa d \hat{B} / d \kappa=-i \kappa \cos \tau \hat{B}
$$

at leading order, and this may be solved exactly by the method of characteristics. Note also that $k_{\omega} \omega^{\prime} / \omega_{w}=0\left[\left(k_{w} / k\right)^{1 / 2}\right] \ll 1$.

The characteristics are defined by

$$
-d \tau=d \kappa / \delta \kappa \sin \tau=d \hat{B} / i \kappa \cos \tau \hat{B},
$$

and the solution is found to be

$$
\hat{B}(\kappa, \tau)=\mathbf{B}\left(\kappa e^{\delta-\delta \cos \tau}\right) \exp \left[-i \kappa e^{-\delta \cos \tau} \int_{0}^{\tau} \cos u e^{\delta \cos u} d u\right]
$$

where $\mathbb{B}(\kappa)=\hat{B}(k, 0)$, the initial wave spectrum.

Since $\delta$ is small, a consistent approximation is

$$
\hat{B}(\kappa, \tau)=\mathbf{B}[\kappa(1+\delta-\delta \cos \tau)] \exp \left[-i \kappa \sin \tau-\frac{1}{2} i \kappa \delta+i \frac{1}{4} \kappa \delta \sin 2 \tau+\cdots\right]
$$

Note that $\kappa \cong \delta k / k_{w} \cong a_{w} k$ need not be assumed small. Notice, too, that other $0(\kappa \delta)$ terms may be dropped while retaining the $\kappa \delta$-terms in the arguments of $\mathbf{B}$ since $\hat{B}(k, 0)$ varies more rapidly with $k$, over the bandwidth $\Delta$; this is equivalent to assuming that $a_{w} \Delta$ is small as well as $a_{w} k_{w}$.

From (2.10), the leading-order free-surface displacement is found to be

$$
\begin{aligned}
\eta(x, t) & =a_{w} \cos \left(k_{w} x-\omega_{w} t+\theta_{w}\right)+\frac{1}{\pi} \int_{0}^{\infty} F(k, \tau) \cos [k x-\omega t-\kappa \sin \tau+\phi] d k \\
F(k, \tau) & \equiv(k / 2 \omega)^{1 / 2}|\mathbf{B}[\kappa(1+\delta-\delta \cos \tau)]| \\
\phi(k, \tau) & \equiv \operatorname{ph} \mathbf{B}[\kappa(1+\delta-\delta \cos \tau)] .
\end{aligned}
$$


The integral need only be taken over positive values of $k$ (in fact, just the bandwidth centred on $k_{0}$ ) since contributions from $k<0$ involve only the smaller, rapidly-oscillating terms neglected from (4.2) above. Comparison with the case $a_{w}=0$, when no long wave is present, shows that each short-wave component of wavenumber $k$ undergoes a periodic frequency modulation, to

$$
\omega(k)+d / d t(\kappa \sin \tau)=\omega(k)+a_{w} \omega_{w} k \cos \tau
$$

also, that the amplitude and phase of each component are related through $F(k, \tau)$ to that of a different wavenumber,

$$
k^{\prime}=k[1-\delta(\cos \tau-1)],
$$

of the initial spectrum $B(k, 0)$. Recall, however, that the omitted next-order terms may also affect the wave amplitude, as in the discrete case considered above.

To demonstrate these results, the particular initial spectrum

$$
(k / 2 \omega)^{1 / 2} B(k, 0)=K \exp \left[-\left(k-k_{0}\right)^{2} / \Delta^{2}\right]
$$

is now considered, where $K$ is a constant that may be taken as real without loss. With no long wave, this spectrum represents a localised wave-packet with displacement

$$
\eta(x, t)=\Delta K \pi^{-1 / 2} \exp \left\{-\frac{1}{4} \Delta^{2}\left(x-\omega_{0}^{\prime} t\right)^{2}\right\} \cos \left(k_{0} x-\omega_{0} t\right) .
$$

Here, $\omega_{0}^{\prime}=d \omega / d k$ at $k_{0}$ is the group velocity of the wave packet.

Application of the saddle-point method to (4.8)-(4.9) shows that the presence of the long wave modifies the displacement to

$$
\begin{aligned}
\eta(x, t)= & a_{w} \cos \left(k_{w} x-\omega_{w} t+\theta_{w}\right) \\
& +\mathrm{a} \exp \left\{-\frac{1}{4} \Delta^{2} r\left(x-\omega_{0}^{\prime} t+\xi\right)^{2}\right\} \cos \left(k_{0} x-\omega_{0} t+\varsigma\right), \\
\xi \equiv & -\left(\kappa / k_{0}\right) \sin \tau, \quad \varsigma \equiv-\kappa \sin \tau+\delta k_{0}(\cos \tau-1)\left(x-\omega_{0}^{\prime} t\right), \\
\kappa= & \kappa\left(k_{0}\right), \quad \mathrm{a} \equiv\left(\Delta K / \pi^{1 / 2}\right)[1+0(\delta)], \quad r \equiv 1-2 \delta(1-\cos \tau) .
\end{aligned}
$$

The local wavenumber and frequency at any instant are then found to be

$$
\begin{aligned}
\hat{k}=k_{0}+\partial_{\zeta} / \partial x= & k_{0}+\delta k_{0}(\cos \tau-1), \\
\hat{\omega}=\omega_{0}-\partial_{\zeta} / \partial t= & \omega_{0}+a_{w} \omega_{w} k_{0} \cos \tau+\delta k_{0} \omega_{0}^{\prime}(\cos \tau-1) \\
& +a_{w} k_{w} \omega_{w} k_{0}\left(x-\omega_{0}^{\prime} t\right) \sin \tau .
\end{aligned}
$$

In the latter, note that the second term exceeds the third and fourth within the packet; but retention of the latter terms is necessary to ensure 'conservation of waves' according to $\partial \hat{k} / \partial t+\partial \hat{\omega} / \partial x=0$. The centre of the wave-packet is at

$$
x=x_{c}(t)=\omega_{0}^{\prime} t+\left(\kappa / k_{0}\right) \sin \tau
$$


and the 'length' of the packet changes with time by the factor $[1+\delta(1-\cos \tau)]$. Since $k_{w} \ll \Delta \ll k_{0}$, the packet length $0\left(\Delta^{-1}\right)$ is short compared with the long wavelength.

It follows from (4.13) that, at leading order, the centre of the packet progresses with a horizontal velocity equal to the sum of the group velocity $\omega_{0}^{\prime}$ and the horizontal orbital-velocity component of the long wave, evaluated at the surface. This is as expected, and agrees with Grimshaw's [4] result (3.22).

There will also be a corresponding $O(\delta)$ change in the amplitude at the centre of the packet, not given at this level of approximation but derivable from the wave action equation, given the local wavenumber, as done by Grimshaw [4].

It is instructive to re-express $\varsigma$ in the form

$$
\varsigma=-\kappa \sin \left(\omega_{w} t-k_{w} x-\theta_{w}\right)-\delta k_{0}\left(x-\omega_{0}^{\prime} t\right),
$$

on using the fact that, within the packet,

$$
\left(\delta k_{0} / \kappa\right)\left(x-\omega_{0}^{\prime} t\right) \cong \sin \left[k_{w}\left(x-\omega_{0}^{\prime} t\right)\right] \ll 1 .
$$

It follows that

$$
\begin{gathered}
\cos \left(k_{0} x-\omega_{0} t+\varsigma\right)=\cos \left[k_{1} x-\omega_{1} t+\kappa \sin \left(k_{w} x-\omega_{w} t+\theta_{w}\right)\right], \\
k_{1} \equiv k_{0}(1-\delta), \quad \omega_{1} \equiv \omega\left(k_{1}\right) .
\end{gathered}
$$

Accordingly, the short waves have averaged wavenumber $k_{1}$ and frequency $\omega_{1}$ and are modulated by the long waves through the $\kappa$-term precisely in agreement with (3.6) and the results of ray theory (Grimshaw [4]).

\section{Conclusion}

The interaction of short gravity waves with a long-wave swell has been considered from a spectral viewpoint that employs Zakharov's [7] formulation. For a discrete train of short waves, results agree with those of Longuet-Higgins and Stewart [5]. Since the latter authors' analysis is strictly valid only for wavenumbers that are comparable in magnitude, it had been conjectured that their results might not apply to interaction of very long and short gravity waves; this agreement is therefore reassuring.

For the interaction of long waves with a continuous spectrum of short waves, such as occurs with a finite wave packet, the present spectral formulation yields new results that accord with Grimshaw's [4] parallel analysis via a wave-action approach; this, too, is satisfying.

However, the spectral approach turns out to be considerably more complicated in application than that using wave-action concepts. Whereas the wave-action 
approach may be applied, with no great additional complication, to capillarygravity waves in finite depth and to long waves that are both large and steep, such extension of the spectral formulation is much less easy (and, for steep waves, impossible). Higher-order approximations in $k_{w} / k$, and extension to include finite water depth and surface tension could certainly be achieved, but a fresh analysis incorporating the separation of long and short length-scales $a b$ initio would be preferable to reduction of the general Zakharov formulation, with its lengthy interaction coefficients, as was done here.

It is only for broad short-wave spectra modified by swell that the present approach is likely to prove advantageous. Then, wave-action concepts break down, since there need no longer be a dominant local wavenumber and frequency with which to work. Though the present analysis was restricted to fairly narrow spectra, the methodology for tackling such problems is now established.

\section{Acknowledgement}

This work was initiated during a visit to Australia in 1986, supported by a grant from the Science and Engineering Research Council which is gratefully acknowledged. I am also indebted to Professor R. Grimshaw and his colleagues at the University of New South Wales for kind hospitality during that time. I am particularly glad to acknowledge stimulating discussions with Professor Grimshaw about this and his own parallel study described in the preceding paper; also to Dr. A. McEwan (C.S.I.R.O. Hobart) and Professor I. Jones (University of Sydney) for remarks that helped stimulate my interest in this topic.

\section{References}

[1] A. D. D. Craik, 'Wave Interactions and Fluid Flows', (Cambridge Univ. Press, 1986).

[2] D. R. Crawford, P. G. Saffman, and H. C. Yuen, "Evolution of a random inhomogeneous field of nonlinear deep-water gravity waves", Wave Motion 2 (1980), 1-16.

[3] D. R. Crawford, B. N. Lake, P. G. Saffman, and H. C. Yuen, "Stability of weakly nonlinear deep-water waves in two and three dimensions", J. Fluid Mech. 105 (1981), 177-191.

[4] R. Grimshaw, "The modulation of short gravity waves by long waves or currents", $J$. Austral. Math. Soc. Ser, B 29 (1988), 410-429.

[5] M. S. Longuet-Higgins, and R. W. Stewart, "Changes in the form of short gravity waves on long waves and tidal currents", J. Fluid Mech. 8 (1960), 565-583.

[6] H. C. Yuen, and B. M. Lake, "Nonlinear dynamics of deep-water gravity waves", $A d v$. in Appl. Mech. 22 (1982), 67-229.

[7] V. E. Zakharov, "Stability of periodic waves of finite amplitude on the surface of a deep fluid", J. Appl. Mech. Tech. Phys. 2 (1968), 190-194. [Translation of Zh. Prikl. Mekh. i. Tekhn. Fiz. 9 (2), 86-94]. 